\title{
Cáncer oculto de mama: reporte de un caso y revisión de la literatura
}

\section{Occult breast cancer: report of a case and review of the literature}

Yesika J. Dávila-Zablah*, Blanca K. Kanagusico-Elguezabal y Margarita L. Garza-Montemayor

Especialidad en Imagen de Mama, Centro de Imagen Diagnóstica, Hospital San José, Monterrey, Nuevo León, México

\section{RESUMEN}

El presente artículo reporta el caso de una paciente de 62 años de edad con un ganglio axilar palpable, con confirmación histopatológica de adenocarcinoma metastásico de origen mamario y con imágenes que confirman la ausencia de tumor primario de mama. Debido a su baja incidencia, se decide presentar el caso, así como una revisión de la literatura.

Palabras clave: Carcinoma oculto de mama. Metástasis axilares. Receptores hormonales. Cáncer.

\section{ABSTRACT}

We report the case of a 62-year-old patient with a palpable axillary node, secondary to occult carcinoma of the breast. The diagnosis was reached after multiple imaging studies and histopathological confirmation. Due to their low incidence, we decided to file the case, as well as review of the literature.

Key words: Occult breast cancer. Axillary nodal metastasis. Hormone receptors. Cancer. 


\section{INTRODUCCIÓN}

El cáncer de mama es la primera causa de muerte por cáncer en la mujer, con una incidencia que ha ido en aumento constante en los últimos años y actualmente representa un problema mundial en los sistemas de salud. El cáncer oculto de mama se define como aquella neoplasia que expresa con adenopatía metastásica, sin manifestación clínica ni radiológica del tumor primario en la mama. Representa el 0.3 al 1\% de todos los cánceres mamarios, por lo que es un reto diagnóstico y un problema terapéutico. La conducta quirúrgica actual continúa siendo controversial debido a su poca frecuencia.

\section{CASO CLÍNICO}

Mujer de 62 años que acude al Centro de Mama del Hospital San José por un área palpable, móvil, localizada en la región axilar izquierda. Cuenta con historia de colocación de implantes mamarios en dos ocasiones (2003 y 2014). Se realizan proyecciones convencionales de mamografía, tomosíntesis y proyecciones con maniobra de Eklund, observando el tejido mamario heterogéneamente denso (patrón c del American College of Radiology, 2013), sin masas, asimetrías, zonas de distorsión ni calcificaciones sospechosas de malignidad (Fig. 1 A y B). Por ultrasonido se identifica en la región axilar izquierda ganglios anormales; el ganglio clínicamente palpable mide $1.5 \mathrm{~cm}$, es irregular, hipoecoico, con pérdida del hilio graso, y a la aplicación del Doppler color muestra vascularidad aumentada (Fig. 2 A). Existen otros seis ganglios anormales, con pérdida del hilio graso y

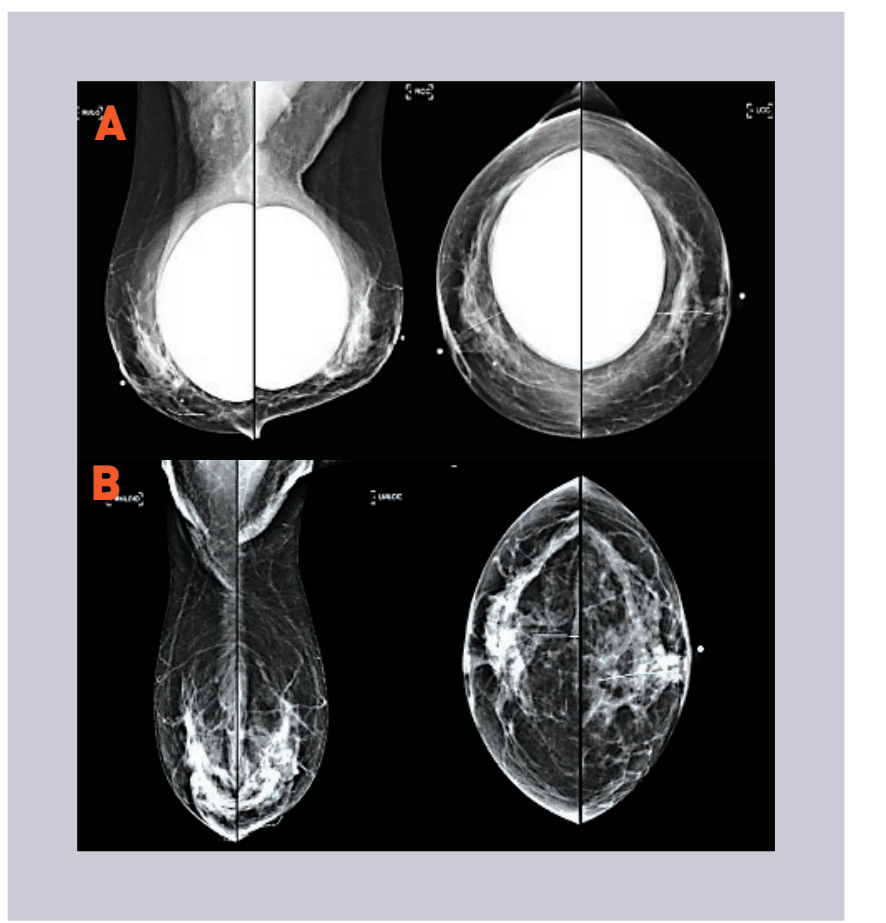

Figura 1. A: proyecciones convencionales de mamografía, observando el tejido mamario heterogéneamente denso.

B: proyecciones con maniobra de Eklund; no hay masas, asimetrías, zonas de distorsión ni calcificaciones sospechosas de malignidad.

de morfología (Fig. 2 B). Se clasifica según el reporte imagenológico mamario y sistema de datos 4, y se recomienda biopsia del ganglio palpable. Posteriormente se realiza biopsia por aspiración con aguja fina, con resultado positivo para células neoplásicas (Fig. 3). Se realiza resonancia magnética y se observa en la región axilar un nódulo sólido irregular, hipercaptante, de bordes microlobulados, que mide $1.5 \mathrm{~cm}$ y corresponde con el ganglio visto por ultrasonido, así como otros ganglios confluentes anormales con engrosamiento cortical, algunos irregulares (Fig. 4). Se realizan nuevamente dos biopsias, una con aguja de corte y otra con aguja fina, y se obtiene resultado de ganglios linfáticos con metástasis de carcinoma, sin presencia de lobulillos ni conductos mamarios (Fig. 5 A y B). Se 


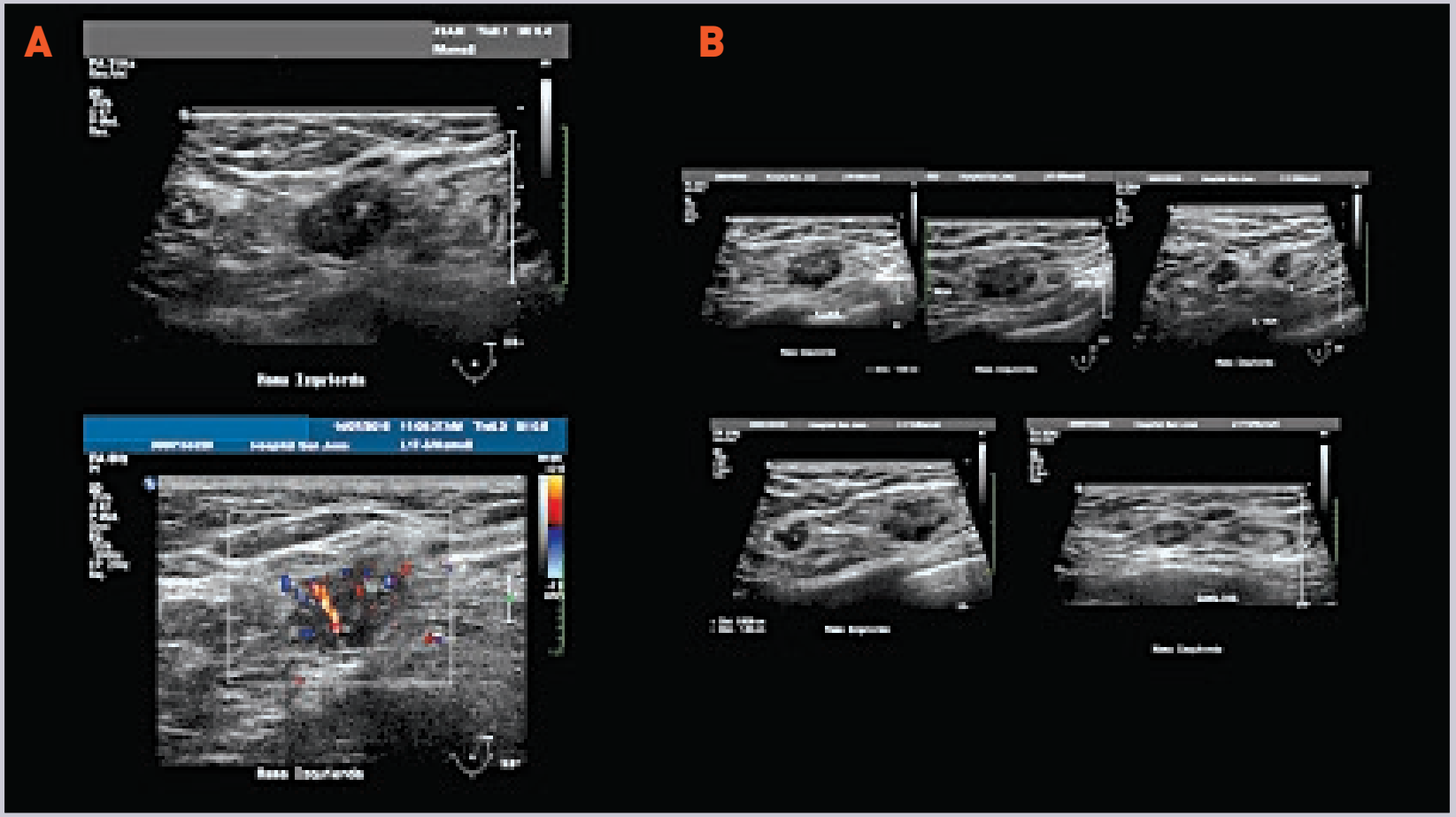

Figura 2. A: ultrasonido axilar izquierdo, en el que se observa un ganglio de $1.5 \mathrm{~cm}$, irregular, hipoecoico, con pérdida del hilio graso, y a la aplicación de Doppler color con vascularidad aumentada. B: existen otros seis ganglios anormales, con pérdida del hilio graso y de morfología.

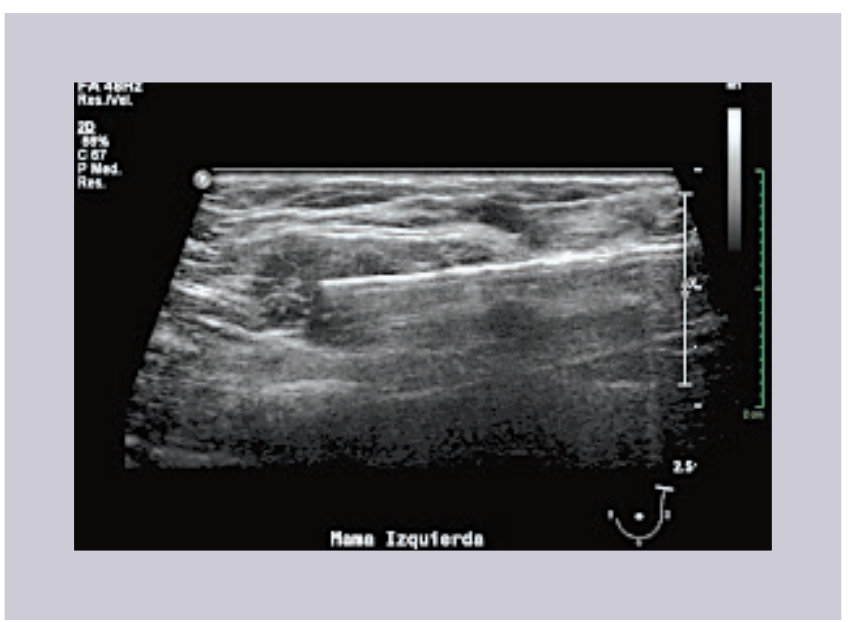

Figura 3. Biopsia de aspiración con aguja fina con reporte positivo para células neoplásicas.

solicita tomografía por emisión de positrones, en la que se visualiza un foco hipermetabólico en la axila izquierda, a nivel del ganglio axilar aumentado de tamaño. No hay evidencia

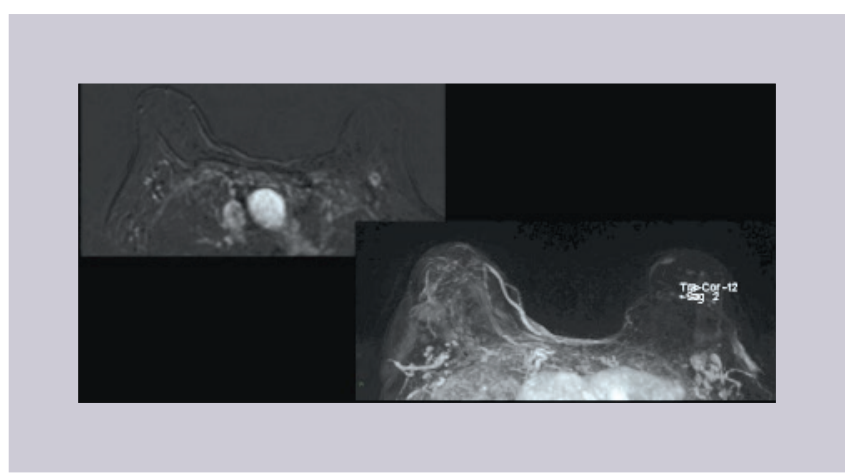

Figura 4. Resonancia magnética que muestra en la región axilar un nódulo sólido irregular, hipercaptante, de bordes microlobulados, que mide $1.5 \mathrm{~cm}$, así como otros ganglios confluentes anormales.

de enfermedad metastásica (Fig. 6). Se realizó biopsia escisional, que tuvo como resultado histopatológico siete ganglios positivos, algunos con rotura capsular e invasión al tejido 


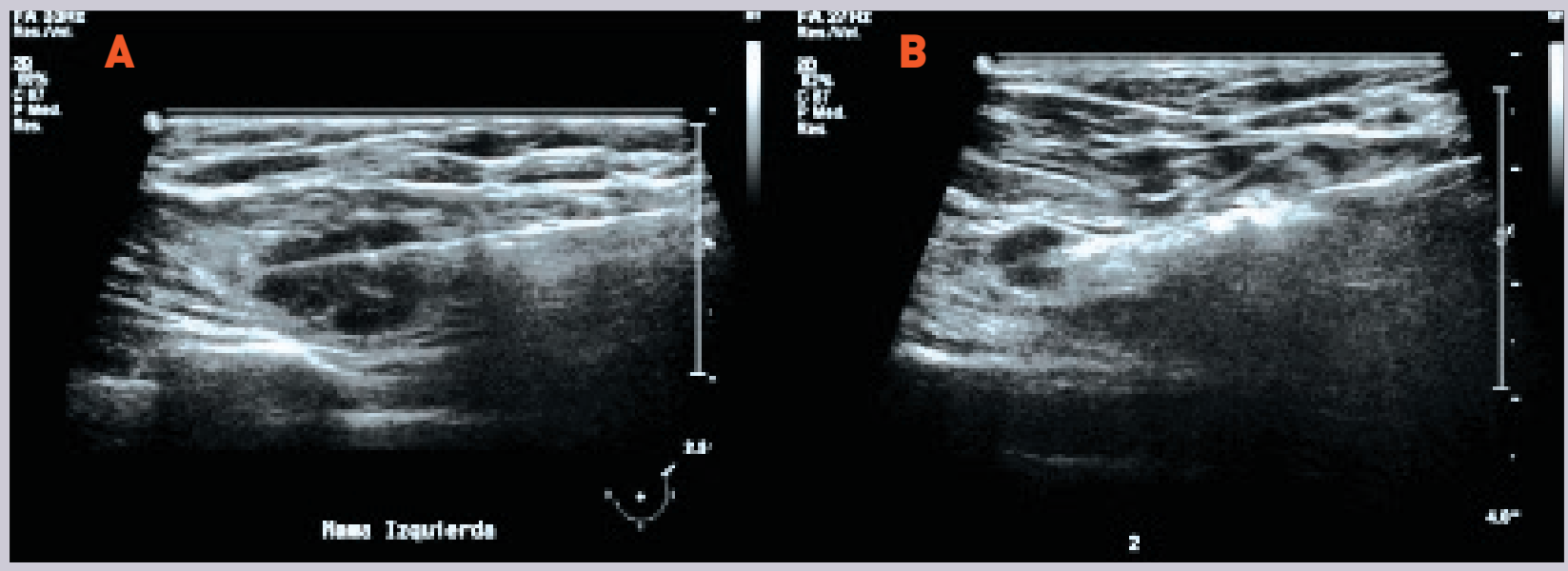

Figura 5. A: biopsia con aguja de corte con resultado de ganglios linfáticos con metástasis de carcinoma, sin presencia de lobulillos ni conductos mamarios. B: Biopsia por aspiración con aguja fina, con el mismo reporte histopatológico.

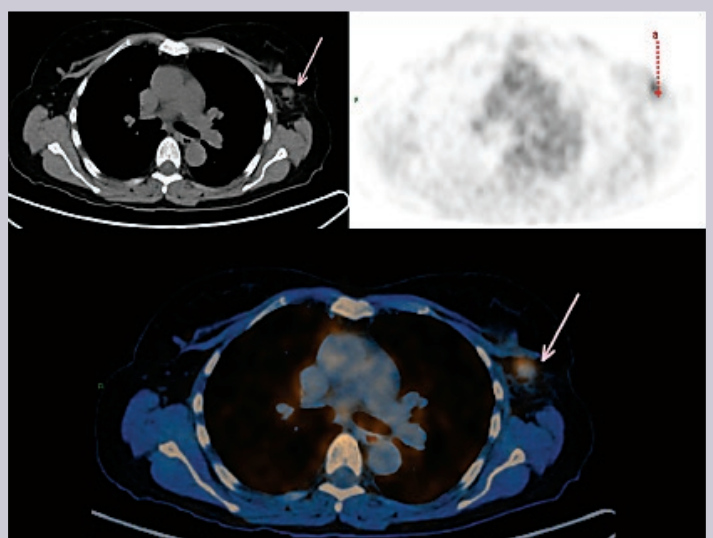

Figura 6. Tomografía por emisión de positrones que muestra un foco hipermetabólico en la axila izquierda. No hay evidencia de enfermedad metastásica.

adiposo; en ninguno de los cortes se identifica tejido mamario. Los receptores de estrógeno y progesterona son negativos; receptor 2 de factor de crecimiento epidérmico humano +++ ; proteína 15 líquida de enfermedad quística y mamoglobina positivas. Resultados compatibles con ganglios metastásicos con origen en la glándula mamaria (Fig. 7).

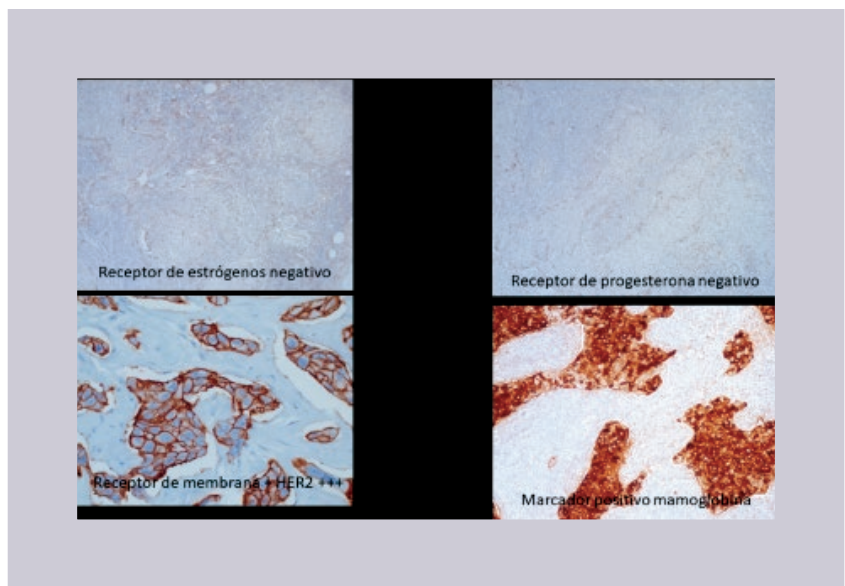

Figura 7. Estudio histopatológico que muestra ganglios positivos, sin identificar tejido mamario, con receptores de estrógeno y progesterona negativos, y con receptor 2 de factor de crecimiento epidérmico humano, proteína 15 líquida de enfermedad quística y mamoglobina positivos.

\section{DISCUSIÓN}

El cáncer oculto de mama constituye una patología poco frecuente. Se define actualmente como aquella neoplasia que se manifiesta con adenopatía homolateral metastásica de un adenocarcinoma histológicamente comprobado, 
sin evidencia clínica ni radiológica de tumor primario en la mama ${ }^{1-18}$.

Esté término fue utilizado por primera vez por Halsted, en 1907, al reportar tres casos de pacientes con ganglios linfáticos metastásicos y glándulas mamarias normales por clínica. Dio seguimiento a las pacientes tras la resección axilar y a los pocos meses se manifestó el cáncer de mama de manera evidente $e^{1,3,8-10,12-16}$. Dos años más tarde, Cameron reportó tres casos similares ${ }^{10}$.

En 1954, Owen intentó estimar la incidencia de cánceres ocultos y encontró 25 casos en 5451 pacientes $(0.4 \%)^{13}$. En estos casos, el método utilizado para el diagnóstico de los tumores de la mama fue tan solo la observación y la palpación ${ }^{1,3,8-10,12-16}$. Posterior a la introducción de la mamografía como método de elección para el diagnóstico del cáncer de mama, la definición cambió a la actualmente conocida ${ }^{3}$.

A pesar del advenimiento de esta modalidad, al revisar la literatura no ha variado mucho la incidencia de esta enfermedad: antes de utilizar la mamografía era del 0.3 al $1 \%^{3}$ y actualmente es del 0.3 al $0.8 \%$ en mujeres ${ }^{1-3,5-12,14-18}$.

Cuando se detecta metástasis axilar, en un 90\% su origen es de cáncer de mama, aunque deben considerarse otras lesiones primarias, entre ellas melanoma, cáncer de pulmón, cáncer de tiroides, adenocarcinoma gastrointestinal (colon, recto), cáncer de páncreas, carcinoma cervicouterino, carcinoma de ovario, cáncer renal y linfoma $a^{1-5,7-12,14-18}$.

En caso de encontrar un cáncer extramamario que cause la adenopatía, ya no se le llama cáncer oculto ${ }^{2}$. Algo que se debe considerar al inicio del estudio en una paciente con sospecha de cáncer oculto es que el origen del carcinoma puede ser la cola de la mama, la axila o tejido mamario ectópico axilar, lo cual puede dilucidarse generalmente con las modalidades estándar para el estudio de la mama ${ }^{4,9,18}$.

El cáncer oculto de mama se presenta con más frecuencia en mujeres, con una edad media al diagnóstico reportada en la literatura de 52 años ${ }^{2}$. Se han reportado algunos casos en hombres $^{7,9}$. La presentación clínica típica es de una masa axilar palpable, que puede medir de 3 a $5 \mathrm{~cm}^{10,11,18}$; se ha reportado un caso con adenopatía supraclavicular izquierda ${ }^{6}$.

Dentro del protocolo de estudio deben incluirse mamografía, ultrasonido de mama y resonancia magnética para determinar la lesión primaria $^{1-4,10,13,16,18}$. La sensibilidad de los estudios para el tumor primario es del 52 al 78\% para la mamografía y del $73 \%$ para el ultrasonido $^{3,8,9,11,12}$.

El método más sensible para analizar la estructura anatómica en pacientes con adenopatía axilar de la mama es la resonancia magnética, que detecta el tumor primario en un $59 \%{ }^{2}$, un $70 \% 1$ y hasta un $85-100 \% \%^{6,14,19}$ de los casos, según los estudios. En la actualidad se utilizan otros métodos de imagen, como la gammagrafía mamaria y la tomografía por emisión de positrones, que miden la actividad maligna celular y pueden detectar tumores de 1 a $3 \mathrm{~mm}$ de diámetro ${ }^{1,3,6,8,10,11,14}$. La tomografía por emisión de positrones permite además la localización anatómica, que tiene como ventaja pocos falsos positivos y la detección de lesiones a distancia, con una sensibilidad del $90 \% \%^{3,6,8,9,11,12}$. 
El examen histopatológico de los ganglios axilares es crítico para su diagnóstico. Se deben incluir pruebas de inmunohistoquímica, con receptores hormonales, que confirmen el cáncer de mama1,3,5,7,10-12. Las células del cáncer de mama expresan comúnmente varios marcadores, como las citoqueratinas, el antígeno carcinoembriogénico, el Ca 15.3, el receptor del factor de crecimiento epidérmico y el receptor 2 del factor de crecimiento epidérmico humano 6,7,9,15; todos ellos presentan una sensibilidad $\mathrm{y}$ una especificidad insuficientes para detectar células tumorales malignas diseminadas provenientes del carcinoma mamario ${ }^{6}$.

Los receptores hormonales positivos serían altamente sugestivos del origen en un cáncer homolateral. El 50\% de las pacientes con cáncer oculto presentan resultados positivos de estrógeno y progesterona ${ }^{8,18}$. Se reporta una sensibilidad y una especificidad de los receptores de estrógenos de 0.63 y 0.95 , respectivamente, con valores menores para los receptores de progesterona ${ }^{5,8,10}$. Otra proteína de gran importancia diagnóstica en este caso es la mamoglobina, que es una proteína de la familia de las secretobinas que se encuentra en el tejido mamario normal y se sobreexpresa en todos los tipos de cáncer de mama; es el marcador de cáncer de mama más estudiado después de la citoqueratina 196,16.

Se reporta también el uso de proteína 15 líquida de enfermedad quística, también conocida como BRST-2, como marcador de diferenciación apocrina, con una sensibilidad y una especificidad de 0.98 y 0.62, respectivamente. Bhargava, et al. ${ }^{20}$ reportan una sensibilidad de la mamoglobina mayor que la de la proteína 15 líquida de enfermedad quística ${ }^{8}$. Sumando la combinación de proteína 15 líquida de enfermedad quística con receptores de estrógeno y progesterona, la sensibilidad es de 0.83 y la especificidad es de 0.85 para carcinomas de mama ${ }^{5,8}$.

El examen histopatológico de la mastectomía revela un 33\% 3,8,9,12,14-16 a un $72 \%$ de los cánceres invasores ocultos de mama ${ }^{2,4-6,18}$, y un $5.8 \%^{4}$ a un $20 \%{ }^{14}$ de cánceres ductales in situ ${ }^{4}$. En la clasificación TNM se estadifica como T0, N1-2, Mx, correspondiendo a un estadio IIA a IIIA de la enfermedad, dependiendo de la afectación ganglionar 9,10,12,14,18. La complejidad del manejo clínico-quirúrgico de esta enfermedad requiere un equipo multidisciplinario ${ }^{1}$. A pesar de los avances científicos y de las numerosas reuniones de expertos en todo el mundo, aún existen dudas en lo que respecta al manejo de la axila, la necesidad de tratamiento quirúrgico o de radiación de la mama, la quimioterapia y la hormonoterapia, debido a su presentación poco frecuente ${ }^{4}$.

El tratamiento tradicional ha sido la mastectomía radical modificada $5,8,10,11,13-16,18$, recomendada en 1909 por Cameron ${ }^{9}$. Sin embargo, en los estudios recientes la mastectomía no aporta mejoría en la supervivencia ni en el control local de la enfermedad 6,8,12. Actualmente, la mastectomía no está recomendada9. La cuadrantectomía se basa en que la localización más frecuente de los tumores primarios es el cuadrante superoexterno. Algunos grupos de trabajo, sobre todos los europeos, sugieren la cuadrantectomía como opción quirúrgica ${ }^{14}$.

En caso de realizar cirugía conservadora, debe complementarse con radioterapia en la axila. Se ha visto que es una buena opción terapéutica, reduciendo el riesgo de recurrencia de la enfermedad, además de obtener resultados estéticos satisfactorios ${ }^{1-6,8,9,12,14,15}$. 
El estándar actual sugiere que, frente a una adenopatía metastásica de origen mamario, sin otro tumor primario demostrable, se debería realizar vaciamiento axilar como forma de proveer factores pronósticos (número de ganglios involucrados, nivel de estos y estudios de receptores hormonales), además de que representa una excelente forma de obtener el control local de la enfermedad de la axila ${ }^{1-6,8,9,12,14,15}$.

Las guías quirúrgicas de la Red Nacional Comprensiva sobre el Cáncer (National Cancer Comprehensive Network) recomiendan realizar mastectomía con disección ganglionar o conservación de la mama y disección ganglionar axilar, seguida de radiación a la mama $^{21}$. El número de ganglios comprometidos se mantiene como el factor más importante de sobreviva, a pesar del modo de presentación y del tratamiento quirúrgico. Adicionalmente se sugiere agregar hormonoterapia, quimioterapia adyuvante o ambas, de acuerdo con el tratamiento estándar 1-3,9-11.

El pronóstico de sobrevida de estas pacientes es del $72 \%$ a 5 años; sin embargo, el involucro de los ganglios y el estado de los receptores pueden dar mejor variables de pronósti$\mathrm{Co}^{2,10,15}$. El pronóstico de sobreviva a 10 años es del 50 al 71\% $\%^{9,11,15}$.

\section{CONCLUSIÓN}

Ante un ganglio palpable anormal recomendamos realizar mamografía, ultrasonido y resonancia magnética para descartar su origen mamario. Cuando se confirma histológicamente como metastásico, debemos considerar otros orígenes de cáncer primario, como melanoma, pulmón, linfoma, tiroides, páncreas, tracto urogenital, linfoma y gastrointestinal; sin embargo, el diagnóstico más probable en un 90\% de los casos corresponde al cáncer de mama. Al sospechar un cáncer oculto de mama debe descartarse con estudios de imagen que el carcinoma sea originado de la prolongación axilar o de tejido mamario ectópico. El examen histológico de los ganglios linfáticos axilares es crítico para el diagnóstico, y se deben incluir pruebas inmunohistoquímicas. Los receptores hormonales positivos son altamente sugestivos del origen mamario. Otras proteínas de gran importancia son la mamoglobina y la proteína 15 líquida de enfermedad quística, que se sobreexpresan en todos los tipos de cáncer de mama.

\section{RESPONSABILIDADES ÉTICAS}

Protección de personas y animales. Los autores declaran que para esta investigación no se han realizado experimentos en seres humanos ni en animales.

Confidencialidad de los datos. Los autores declaran que los datos de las pacientes que aparecen en este artículo son anónimos.

\section{Derecho a la privacidad y consentimien-} to informado. Los autores declaran que los datos de las pacientes que aparecen en este artículo son anónimos.

\section{BIBLIOGRAFÍA}

1. Jurado Bambino A, Polit Arguello P, Zambrano Pérez D. Cáncer oculto de mama: caso clínico. Rev Oncol Ecu. 2012;22:53-7.

2. Das DK, Teo ZC, Choudhury U. Occult breast cancer presenting as axillary nodal metastasis - a case report and literature review. International Journal of Collaborative Research on Internal Medicine \& Public Health. 2012;4:1162-6. 
3. Kim Y, Kim H, Cho J, Kwon S, Yeo S, Kang SH. The results of occult breast carcinoma treated with axillary node dissection only: five cases reports. J Breast Dis. 2016;4:24-7.

4. Acosta V, Coutinho M, Contreras A, Ravelo R, Marin CE, Ramírez AK, Carcinoma oculto de la mama. Experiencia en el centro clínico de estereotaxia-CECLINES. Rev Venez Cir 2014;67 (1):5-9.

5. Sanguinetti A, Polistena A, Dermo G, Lucchini R, Triola R, Conti C, Axillary metastases from occult breast cancer. Annali Italiani di Chirurgia. 2014;85:1-3.

6. Arnal A, Moreno A, González I, Martín E, Asensio E, Vázquez F. Manifestación clínica atípica de un cáncer oculto de mama. Revista de Senología y Patología Mamaria. 2013;26:33-7.

7. Mo S, Hui D, Kyung S, Min-Young B, Youn S, Young M, et al. Occult breast cancers manifesting as axillary lymph node metastasis in men: a two-case report. J Breast Cancer. 2012;15:359-63.

8. Oualla K, Elm F, Arifi S, Mellas N, Melhouf A, Bouhafa T, et al. Occult primary breast cancer presenting with axillary nodal metastasis: report of 3 cases. J Clin Gynecol Obstet. 2012;1:85-8.

9. Luna MA, Sánchez-Méndez S, Mariscal A, Viscaya S, Vallejos V, Solá M, et al. Cáncer oculto de la mama. Caso clínico y revisión de la literatura. Clin Invest Gin Obst. 2012;39:21-8.

10. Wang X. Presentation of axillary metastases from occult breast carcinoma. Chinesse Journal of Clinical Oncology. 2007;4:1-5.

11. Mottola J, De RE, Mazoccatto C. Carcinoma primario oculto de la mama. Femina. 2006;34:585-90.
12. Kee K, Shwartz R, Iglesis R. Cáncer de mama oculto: dos casos clínicos analizados según el concepto actual. Rev Med Chile. 2006;134:1166-70.

13. Lloyd M, Nash A. Occult breast cancer. Ann R Coll Surg Engl. 2001;83:420-4.

14. Saure Sarria VM, Cardoso Hernández J, Hernández Herrera L. Cáncer oculto de mama: presentación de caso. Archivo Médico de Camagüey. 2010;14:1-10.

15. Abe H, Naitoh H, Umeda T. Occult breast cancer presenting axillary nodal metastasis: a case report. Jpn J Clin Oncol. 2000;30:185-7.

16. Watson M, Dintzis S, Darrow C, Voss LE, DiPersio J, Jensen R, et al. Mammaglobin expression in primary, metastatic and occult breast cancer. Cancer Res. 1999;59:3028-31.

17. Baker D. Magnetic resonance imaging of occult breast cancer. Clin Breast Cancer. 2000;1:66-7.

18. Harris JR, Lippman ME, Morrow M, Osborne CK. Enfermedades de la mama. Cáncer primario oculto con metástasis axilares. Marban 2009;65: 797-801.

19. Mann R, Kuhl C, Kinkel K, Boetes C. Breast MRI guidelines from the European Society of Breast Imaging. Eur Radiol. 2008;18:1307-18.

20. Bhargava R, Beriwal S, Dabbs DJ. Mammaglobin vs GCDFP-15: an immunohistologic validation survey for sensitivity and specificity. Am J Clin Pathol. 2007;127(1):103-13.

21. Sohn G, Son BH, Lee SJ, Kang EY, Jung SH, Cho SH, et al. Treatment and survival of patients with occult breast cancer with axillary lymph node metastasis : a nationwide retrospective study. J Surg Oncol. 2014;110:270-4. 